\title{
A Study of Mutual Insurance for Bank Deposits
}

\author{
CAROLE BERNARD \\ Ph.D. Student, Teaching Assistant at ISFA, University of Lyon 1, France \\ OLIVIER LE COURTOIS \\ Associate Professor of Finance at EM Lyon, France \\ FRANÇOIS QUITTARD-PINON \\ Professor of Finance at ISFA, University of Lyon 1, France
}

Received July 22, 2004; Revised July 22, 2004

\begin{abstract}
This article displays a study on the mutual insurance of bank deposits. A system where deposits are first insured by a consortium then by the Government is envisaged. We wish to compute the fair premia due to both the consortium and the Government. Various types of covenants aiming at making banks reduce their risks are detailed. These provisions can be, as is the case in Chapter 11, of a Parisian type. This means that surveillance is based on the path followed by the assets or the leverage. We compare these various types of covenants and conclude on the proposal for new regulatory provisions.
\end{abstract}

Key words: parisian options, deposit insurance, surveillance mechanisms, contingent claims analysis

JEL Classification No.: G13, G21

\section{Presentation}

Deposit insurance constitutes one of the most studied problems of financial economics. Deposit insurance policies can be structured very differently across countries. They can be public, private, or semi-public. In the United States, they are managed by a Government agency. In some other countries, like Italy, a consortium of banks is in charge of them. In addition to that, several features make deposit insurance policies differ noticeably. Too risky deposits as well as foreign deposits can be excluded or not from the insurance system. It can be a Government policy to favor more or less deposit insurance, and subsidies will vary in proportion. Coverage can be full or not. Premia are paid ex ante or ex post. Deposit insurance policies are thus multiple in form, and the theoretical tools to valuate them should consequently also be multiple.

Two main approaches to the pricing of deposit guarantees are in competition. In the first approach, deposit guarantees are valued like standard derivatives and the Black and Scholes machinery is strongly involved. Merton [1977, 1978] was the first author to provide a first coherent valuation procedure based on his earlier work (see Merton [1974]) on corporate 
debt pricing, work that is itself a bud of the famous Black and Scholes framework [1973]. Ronn and Verma (1986) then extended Merton's approach to permit efficient calibration of the model on public equity data. In the second approach, deposit guarantees are priced actuarially as premia equal to expected losses. References to this methodology can be found in Laeven [2002] and FDIC [2000]. The idea to calculate an expected loss is basically to compute the product of an expected default probability times a loss given default. Expected default probabilities can be computed from ratings provided by agencies such as Moody's; losses given default can be estimated econometrically from past loss records.

This paper will display a study of mutual or consortium insurance of bank deposits. Such a system has been described by De Giuli, Maggi, and Paris [2003]. These authors value deposit guarantees as sums of optional contributions related to the consortium and the Government. Relying also on options theory and Merton's financial tenets, we will develop on the impact of consortium policies upon deposit institutions behaviors. Covenants of Parisian and cumulative Parisian types will be envisaged; their impact on the premia to the consortium and Government will be computed. We will also introduce a new type of covenant, before concluding. To sum up, the goal of this paper will be to study the introduction, pricing and effects of new covenants in deposit insurance.

\section{A simple model}

In this subsection, we give a simple model allowing for the valuation of deposit insurance premia. We start by explaining our theoretical approach and then we expose the results obtained with this approach.

\subsection{Theoretical approach}

Merton [1978] provides a simple but efficient way to price bank guarantees as put options on the firm assets with a strike price equal to the amount of deposits. We are concerned here with the valuation of guarantees when banks are part of a consortium. De Giuli, Maggi, and Paris [2003] make a study of consortium guarantee valuation in Italy under an optional approach. The model we propose here is related to the above approaches, but simpler than the second one that postulates many underlying determinants.

Let a consortium insure bank deposits up to a given amount $K$ per bank. We suppose that the insurance system is designed such as to make bank deposits completely insured. In other words, if a bank falls short of $H>K$ on its deposits, the consortium will reimburse $K$ to the bank customers and the Government will take care of the reimbursement of the remaining debt shortfall, equal to $H-K$. We assume, contrary to De Giuli, Maggi and Paris [2003], that guarantee premia are paid in advance to the consortium — not upon default of a given bank. In the Italian system, no premia are paid to the Government and the premia paid to the consortium are too low, implying a joint subsidies effect towards deposit institutions. Here, we wish to compute the value of the guarantees or fair premia due to both the consortium and the Government. 
In a model à $l a$ Merton, the final payoff of the put option valuing the bank deposit guarantee writes max $\left(D_{T}-V_{T}, 0\right)$ where $D_{T}$ is the deterministic deposit amount $\left(D_{T}=e^{r T} D_{0}\right), V_{T}$ the bank assets and $r$ the instantaneous interest rate. In such a framework, debt is made of deposits and constitutes the strike. Let it be a one-period model where the maturity $T$ is typically equal to one year. As concerns $V$, we shall assume in all this paper that it can be represented by a geometric Brownian motion with volatility parameter $\sigma$.

Now, let us take into account the cutoff level $K$. The Government will reimburse losses in excess of $K$, idem est $D_{T}-V_{T}-K$. Therefore, the value of the Government guarantee is a put option with final payoff equal to $\max \left(D_{T}-V_{T}-K, 0\right)$. Next, let us consider the value of the consortium guarantee. It is related to the difference between the total amount to be repaid in case of bankruptcy and the amount that would be paid by the Government in excess of $K$ (the consortium starts to reimburse losses up to $K$, then passes on to the Government). Hence, the final payoff of the option related to the consortium expresses as: $\max \left(D_{T}-V_{T}, 0\right)-\max \left(D_{T}-V_{T}-K, 0\right)$. The value of the consortium guarantee is consequently a spread option - spread between two puts.

Thus, we can price a deposit guarantee as a sum of two options - a put option for the Government part, and a put spread option for the consortium part. This is when liabilities are only made of deposits. What happens when senior as well as junior debts are considered? Let there be some senior debt, $S$, strictly prior to the deposits, $D$, and some junior debt, $J$, coming after $S$ and $D$. A formula for the total value of the guarantee can be expressed under the risk-neutral measure $Q$ as:

$$
\frac{D_{0}}{S_{0}+D_{0}+J_{0}} E_{Q}\left[e^{-r T}\left(S_{T}+D_{T}-V_{T}\right)^{+}\right]
$$

where, in case of bankruptcy, part of the assets serve to reimburse senior debt in strict priority.

This formula is simple in essence but does not cover many important empirical features. As mentioned in Shibut [2002], the effects of assuming various capital structures for banks are multiple and contrary in implications. For instance, a bank strongly financed by senior debt (little by deposits) minimizes its gross insurance premium — but the risk is the same for the insurer. One could argue in another direction that financing by senior debt is an indication that a bank has a long-term hence serious debt policy. As concerns junior debt, it is more expensive than senior debt; high coupon payments can mean a higher probability of default. On the contrary, a bank that finances itself by junior debt is under surveillance by the market and this would tend to make managers lead better policies.

Fair valuation of bank guarantees when there exist senior and junior debts is not an easy problem. This is because the risks associated to senior and junior debt can be assessed in many ways, as illustrated in the previous paragraph. Indeed, in such a situation, it seems difficult to build a global assessment methodology of the risk associated to the liabilities of a bank; a bank-by-bank actuarial evaluation would appear more fruitful. In light of this, formula (1) is too simple to incorporate plainly the various effects associated to the capital structure of the bank under study.

Let us now give the fundamental formulae that are going to be used—and extended—in this article. We assume that senior debts are negligible (as is the case in Italy) and that 
the actuarial assessment of the risk of a bank liability structure comes down to a unique parameter $\theta$. This parameter is the proportion of deposits over total liabilities. In Italy, it is typically equal to $1 / 2$. For the sake of simplicity, we will choose $\theta=1$ in our applications. The consortium covers losses up to $K$; the value of the consortium guarantee is denoted by $O_{C}$. The Government covers losses in excess of $K$; the corresponding guarantee value is denoted by $O_{G}$.

We can now give the core formulae:

$$
O_{G}=\theta E_{Q}\left[e^{-r T}\left(D_{T}-V_{T}-K\right)^{+}\right]
$$

and:

$$
O_{C}=\theta\left(E_{Q}\left[e^{-r T}\left(D_{T}-V_{T}\right)^{+}\right]-E_{Q}\left[e^{-r T}\left(D_{T}-V_{T}-K\right)^{+}\right]\right)
$$

which price the Government and consortium guarantees from the point of view of the deposit institution.

\section{2. $\quad$ First results}

We now make an analysis of the premia that a bank ought to pay to the consortium and Government in order to insure its deposits. Let the following parameters be fixed until the end of this subsection:

\begin{tabular}{ccccc}
\hline$V_{0}$ & $\sigma$ & $r$ & $\theta$ & $T$ \\
\hline 100 & 0.25 & 0.03 & 1 & 1 \\
\hline
\end{tabular}

where $V_{0}$ is the initial value of the assets, $\sigma$ their volatility, and $r$ the risk-free instantaneous interest rate.

We plot in figure 1 the premia for $D_{0}$ ranging from 70 to 100 and $K=10$. We observe that when the total amount of deposits is low (the bank is quite safe, having lots of capital in excess) the premium due to the consortium is higher than the one due to the Government. When the amount of deposits is huge and close to the initial value of the assets, the bank is riskier and the probability that the Government needs to reimburse a huge amount in excess of $K$ (a bigger amount than the consortium) is high. Therefore, in such a configuration, the premium to be paid to the Government is higher than the one owed to the consortium, which is confirmed by the figure.

In figure 2, we do the contrary: we plot the premia along $K$ ranging from 5 to 30 with an initial value of the deposits set to $D_{0}=85$. One observes straightforwardly that when $K$ increases, the coverage of the consortium increases with respect to the one of the Government. When a bank defaults, the higher $K$, the higher the contribution from the consortium, hence the higher the premium to the consortium and the lower the one to the Government. 


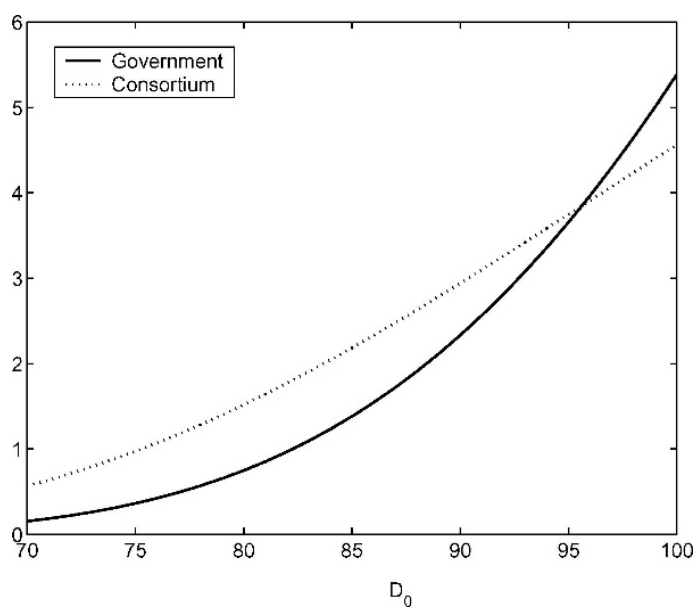

Figure 1. Premia w.r.t. $D_{0}$.

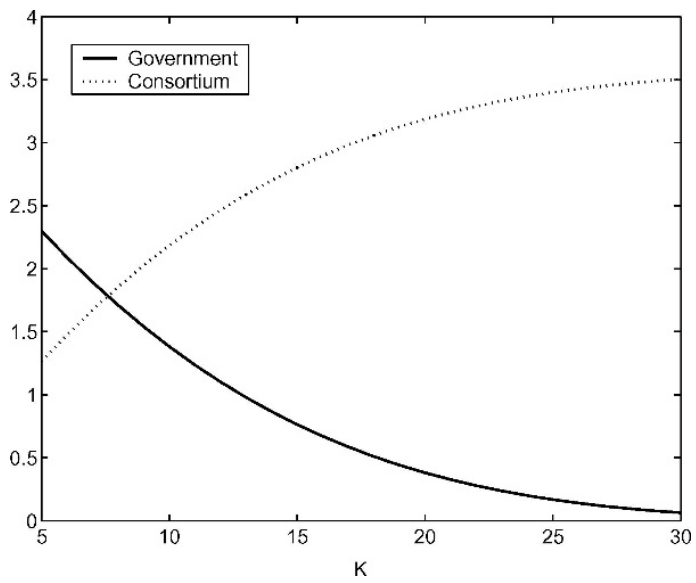

Figure 2. Premia w.r.t. $K$

Then, in figures 3 and 4, we graph the premia due to the Government and consortium separately. These plots are done with respect to $D_{0}$, each curve corresponding to a different value of $K(K=5,10,20,30)$. The higher $K$ is, the lower is the curve on the left graph and the higher it is on the right one. These figures are confirmations of the fact detailed in the previous paragraph: when $K$ increases, the consortium has to pay back a higher quantity of deposits upon default, whilst the Government, relieved by an equivalent amount, has to reimburse fewer deposits. 


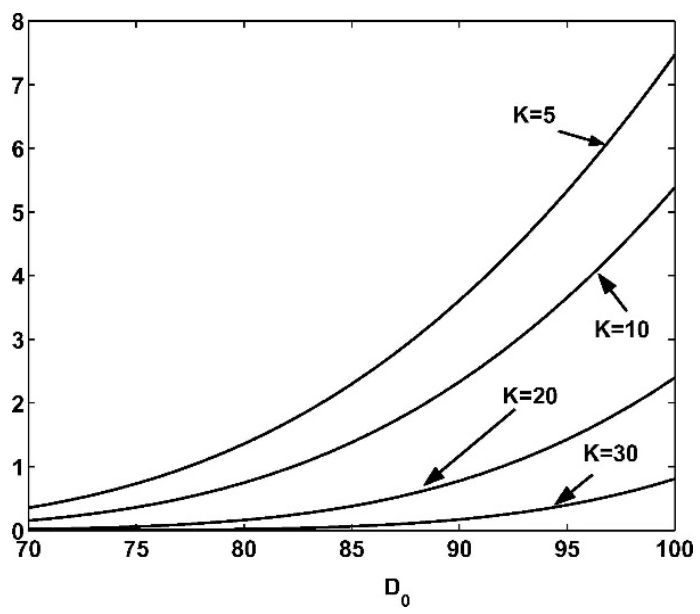

Figure 3. Premia to the government.

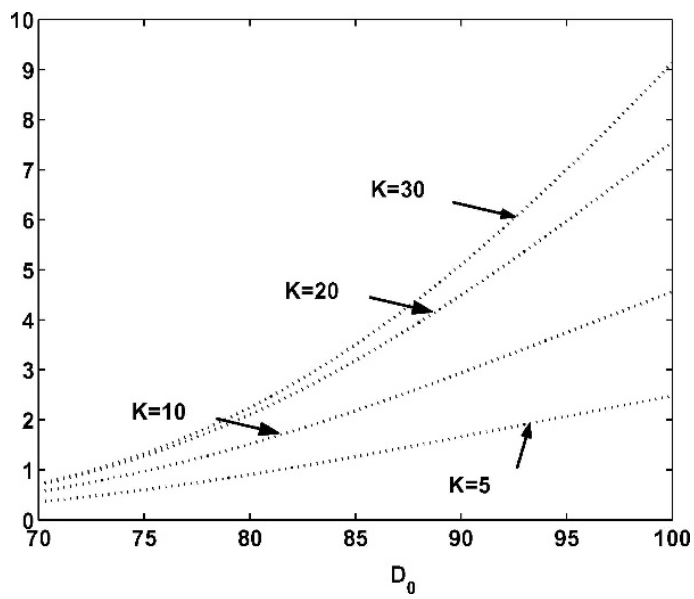

Figure 4. Premia to the consortium.

\section{Introducing a parisian feature}

We detail in this section a new model that allows dealing with covenants designed to protect the consortium and make banks reduce their risks.

\subsection{Motivations for a parisian safety covenant}

Let there be, similarly as in the Italian market, a covenant designed in the following way: when a deposit institution's typical risk process (its leverage, or its assets) remains above or below a given level for a given amount of time, then this bank is excluded from the 
consortium. Note that if this bank goes bankrupt after exclusion, the Government takes care of the whole guarantee. In the particular case of this study, we assume that a bank whose assets have remained more than the prespecified time $d$ below the critical level $L$ will be excluded from the consortium. This type of constraint should incite banks to reduce their risks by fear of being excluded from a good protective system; yet we will see that this might result in dangerous opportunistic behaviors.

The question that arises at this point is: what is the value of the deposit guarantee or premium related to a bank that can be excluded from its consortium in the manner detailed above? Keeping with the valuation tools stemming from options theory, this question can in fact be reformulated as: what kind of option could render the exclusion covenant just defined? The answer to this question has to be sought for in the exotic options literature. Indeed, options written with such covenants are called Parisian options. These options have been introduced and studied by Chesney, Jeanblanc and Yor [1997]. Parisian options are particular kinds of barrier options whose payoff depends on the history of the underlying asset price. The condition on the underlying is that it must remain above or below the Parisian barrier during a prespecified time; this is precisely what we need for our deposit guarantee pricing problem.

Let us now write the formulae giving the premia due to the Government and consortium in the context of a Parisian restriction. The superscript $p$ stands for a Parisian covenant and we write the indicator $\mathbb{1}^{p}$ in the expression of the consortium guarantee $O_{C}^{p}$ to mean that the bank deposits are insured by the consortium only if its assets did not stay more than $d$ below the threshold $L$. The consortium Parisian guarantee is a down and out Parisian put spread. It can be priced by extending (3) as follows:

$$
O_{C}^{p}=\theta e^{-r T} E_{Q}\left[\left(D_{T}-V_{T}\right)^{+} \mathbb{1}^{p}-\left(D_{T}-V_{T}-K\right)^{+} \mathbb{1}^{p}\right]
$$

As for the government Parisian guarantee, it is equal to the standard guarantee plus the contribution that is abandoned by the consortium in case of exclusion; it thus expresses as:

$$
O_{G}^{p}=O_{G}+\left(O_{C}-O_{C}^{p}\right)
$$

Note that immediate recovery of the total guarantee can be done by summing up the two previous formulae:

$$
O_{G}^{p}+O_{C}^{p}=O_{G}+O_{C}=\theta e^{-r T} E_{Q}\left[\left(D_{T}-V_{T}\right)^{+}\right]
$$

Valuing deposit guarantees is thus a matter of valuing Parisian down and out put options. In the coming subsection, we explain how this can be achieved in a simple manner.

\subsection{Valuation of Parisian options and guarantees}

We shall now explain how Parisian options can be priced in the Black and Scholes framework. The material exposed here will prove useful in the coming subsection where pricing of deposit guarantees will be performed. 
In addition to the lognormal underlying $V$, let there be a riskless security-a bondwhose instantaneous interest rate, denoted by $r$, is supposed constant. The market is then complete, and there exists a unique risk-neutral measure $Q$ equivalent to the historical probability measure. Denote by $\sigma$ the underlying's constant volatility, $T$ the option maturity and $K$ the strike. $L$ is the Parisian barrier and $d$ the time lag that has to be spent beyond $L$ to activate or deactivate the option.

Describe the underlying $V$-it will represent the assets of the bank in the applications-by the following stochastic differential equation :

$$
\frac{d V}{V}=(r-q) d t+\sigma d z
$$

where $z$ is a $Q$-Brownian motion, $q$ the continuous dividend rate. Then:

$$
V_{t}=V_{0} e^{\left(r-q-\frac{1}{2} \sigma^{2}\right) t+\sigma z_{t}}=V_{0} e^{\sigma\left(m t+z_{t}\right)}
$$

under the convention:

$$
m=\frac{1}{\sigma}\left(r-q-\frac{\sigma^{2}}{2}\right)
$$

Let $\tau$ represent the time at which the Parisian condition is verified, in other words the moment when the underlying has been continuously more than $d$ below $L$. Using standard risk-neutral arguments, one values a Parisian down and out put option according as:

$$
P_{0}^{d}=e^{-r T} E_{Q}\left[\left(K-V_{T}\right)^{+} 1_{\tau>T}\right]
$$

By definition, the value of a Parisian down and in put option is:

$$
P_{i}^{d}=e^{-r T} E_{Q}\left[\left(K-V_{T}\right)^{+} 1_{\tau<T}\right]
$$

and these two puts are related by the following parity relationship:

$$
P_{o}^{d}+P_{i}^{d}=P\left(V_{0}, T\right)
$$

where $P\left(V_{0}, T\right)$ is the price of a standard put option.

As a conclusion, it appears from formula (6) that to compute Parisian down and out puts, which is necessary in the process of deposit guarantee valuation, it is enough to be able to compute Parisian down and in puts.

When $V_{0}>L$ and $K \leq L$, one has (see Chesney et al. [1997]):

$$
P_{i}^{d}=e^{-\left(r+\frac{1}{2} m^{2}\right) T} \int_{-\infty}^{k} e^{m y}\left(K-V_{0} e^{\sigma y}\right) h_{2}(T, y) d y
$$

where $K=\frac{1}{\sigma} \ln \left(\frac{K}{V_{0}}\right)$ and $l=\frac{1}{\sigma} \ln \left(\frac{L}{V_{0}}\right)$. 
The function $h_{2}(T, y)$ is known through its Laplace transform (w.r.t. T). We denote this transform by $\hat{h} 2$; it admits the closed-form expression:

$$
\begin{aligned}
\hat{h}_{2}(\lambda, y)= & \frac{e^{y \sqrt{2 \lambda}}}{\sqrt{2 \lambda} \Psi(\sqrt{2 \lambda d})}+\frac{\sqrt{2 \pi d} e^{\lambda d}}{\Psi(\sqrt{2 \lambda d})} \times\left[e ^ { y \sqrt { 2 \lambda } } \left(\mathcal{N}\left(-\sqrt{2 \lambda d}-\frac{y-l}{\sqrt{d}}\right)\right.\right. \\
& \left.-\mathcal{N}(-\sqrt{2 \lambda d}))-e^{(2 l-y) \sqrt{2 \lambda}} \mathcal{N}\left(-\sqrt{2 \lambda d}+\frac{y-l}{\sqrt{d}}\right)\right]
\end{aligned}
$$

where $\Psi(z)=1+z \sqrt{2 \pi} e^{\frac{z^{2}}{2}} \mathcal{N}(z)$ and $\mathcal{N}$ is the Gaussian cumulative distribution function.

To sum up, the computation of down and in Parisian puts can be done in two steps. First, one needs to invert the Laplace transform $\hat{h}_{2}$ w.r.t. their first argument so as to get back $h_{2}$. Then, using (7), one should perform a numerical integration. Whilst the second step is a mere quadrature, the first one is by far more involved. In numerical applications, we make use of the method Bernard, Le Courtois and Quittard-Pinon [2005] developed to compute inverse Laplace transforms and price Parisian options.

The parity relationship (6) allows obtaining the Parisian down and out put from the down and in put, and this immediately yields the values of the consortium and Government guarantees by equations (4) and (5). We proceed along these lines in the following in order to lead our numerical analysis.

\subsection{Analysis of the results}

Let us now come to the analysis of bank deposit guarantees under a Parisian covenant. In figures 5 and 6, we compare the standard and Parisian premia. We take $K=20$ and $D_{0}$ ranging from 70 to 100 . The barrier level is set to $L=85$ and the time period determining

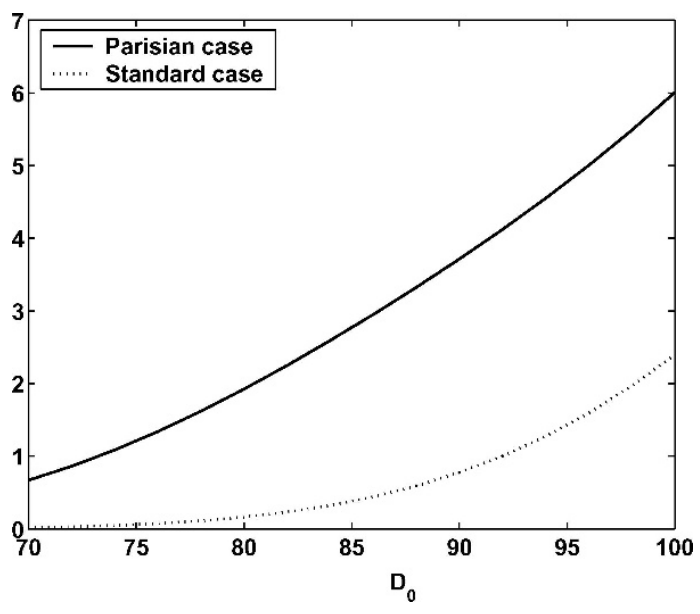

Figure 5. Premia to the Government. 


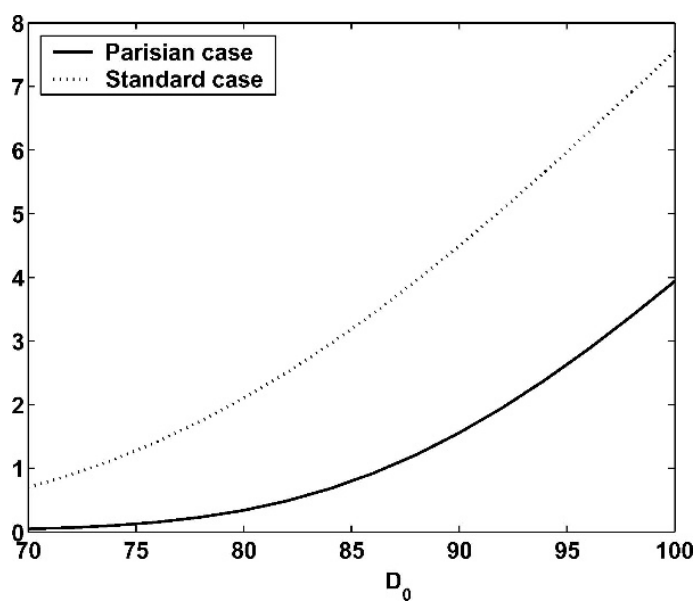

Figure 6. Premia to the consortium.

exclusion to $d=0.2$. In other words, when the assets of the bank under surveillance remain more than $d=0.2$ below $L=85$, this bank is excluded from the consortium, and, in case of bankruptcy, depositors get fully reimbursed by the Government.

We see from these two figures the effect of introducing a Parisian covenant: the premia to the Government are increased whilst the premia to the consortium are lowered. This feature is logical since in some cases deposits become insured only by the Government.

We give more details on the impact of the Parisian covenant in figures 7 and 8 . We set for both plots $K=10$ and $D_{0}=85$. For the first figure, $L=85$ and $d$ is ranging from 0 to

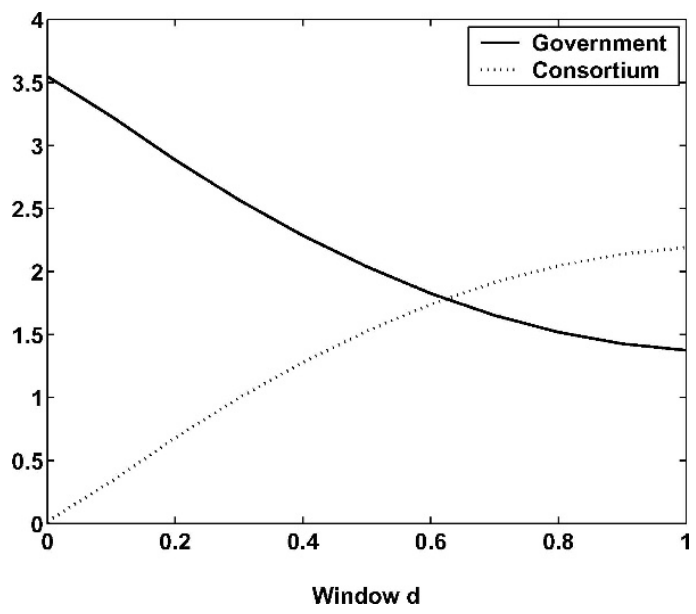

Figure 7. Premia w.r.t. $d$. 


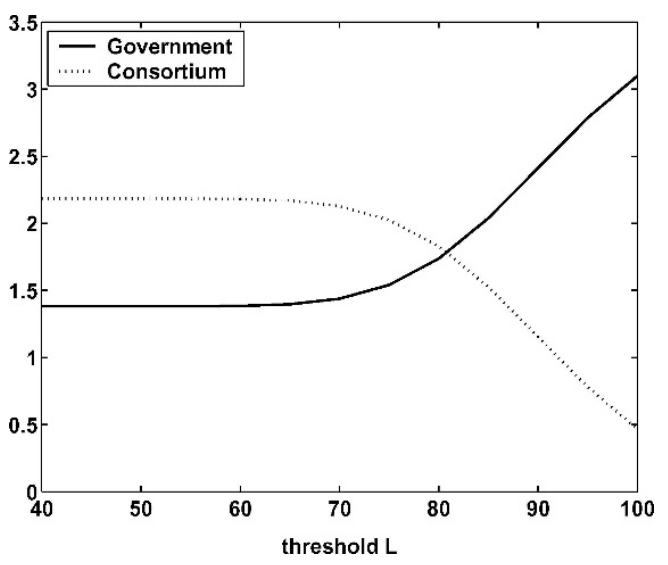

Figure 8. Premia w.r.t. L.

1 ; for the second one $d=0.5$ and $L$ goes from 40 to 100 . In figure 7, we see that when $\mathrm{d}$ increases, the premium to the consortium increases whilst the premium to the Government decreases. This is because for high values of $d$, the probability of deactivation or exclusion is low, which results in a smaller possible contribution from the Government. We observe the same kind of pattern in figure 8 . When the value of the threshold is low, the probability of exclusion becomes null and the Government guarantee is minimized with respect to the consortium one. In the extreme case when $d$ is unitary or $L$ is quite low, we recover the standard case where no exclusion covenant exists. This is confirmed by computation of the Government and consortium premia which are respectively equal to 1.38 and 2.18 in the standard case.

\section{Cumulative versus naive clockworks}

In this section, we study deposit guarantees when surveillance is of a cumulative Parisian type. We start by explaining how these guarantees can be valued before coming to the empirical analysis. This approach is similar to the one given in the preceding section and can be considered complementary material.

\subsection{Pricing cumulative Parisian guarantees}

It is possible to design exclusion covenants in a similar-yet slightly different-way as when constructing Parisian covenants. Suppose assets are monitored with a clock that counts the total time spent below a given threshold. This situation is different from the one encountered in the previous section because we do not impose the assets to stay strictly below the threshold during a continuous period $d$. Instead, a clock is initiated and counts the total time spent below the threshold over the period $T$. If this total time is superior to $d$, the bank is excluded from the consortium. 
When referring to such covenants, we will use the expression 'cumulative Parisian'. Indeed options designed with such covenants exist; they are called cumulative Parisian options and were valued by Hugonnier [1999] and Moraux [2002]. Following the same methodology as the one employed in Section 3, we value cumulative Parisian bank deposit guarantees (the bank can be excluded from the consortium upon no compliance to a cumulative Parisian covenant) by means of cumulative Parisian options. Indeed, for our computations, we will only need to adapt formulae (4) and (5) by considering cumulative Parisian times.

\subsection{Using cumulative Parisian options}

A cumulative Parisian option is activated when the total time spent by the underlying $V$ under a threshold $L$ is greater than a prespecified time span $d$. This option is different from a standard Parisian option because the underlying does not have to stay below or above the level during a strict period $d$, but just to spend a total time $d$ beyond the barrier before maturity $T$.

It appears that the cumulative Parisian options defined and studied by Hugonnier are in options. In options are linked to out options by a standard parity relationship that holds for both Parisian and cumulative Parisian options, see formula (6). This parity relationship permits to value cumulative Parisian out puts, in other words cumulative Parisian guarantees, from cumulative Parisian in put prices. The question at stake is thus now: how do we price cumulative Parisian in puts?

We choose the superscripts ${ }^{+}$or ${ }^{-}$to precise whether the occupation time is considered above or below the level $L$ and we denote by $C$ and $P$ the cumulative Parisian calls and puts. One has the formula:

$$
P^{-}\left(T, V_{0}, K, L, r, q\right)=V_{0} K C^{+}\left(T, 1 / V_{0}, 1 / K, 1 / L, q, r\right)
$$

that relates down and in puts to up and in calls. These calls can be computed as follows (m being defined as in the previous section):

$$
C^{+}\left(T, V_{0}, K, L, r, q\right)=V_{0} \xi(m+\sigma, T, K, L, d)-K \xi(m, T, K, L, d)
$$

where:

$$
\begin{aligned}
\xi(\mu, T, K, L, d)= & \int_{d}^{T} d s\left[\int_{k \wedge l}^{1} e^{\mu x} \Upsilon(2 l-x, 0, s, T-s) d x\right. \\
& \left.+\int_{k \vee l}^{+\infty} e^{\mu x} \Upsilon(l, x-l, s, T-s) d x\right]
\end{aligned}
$$


using the conventions from the previous section on $l$ and $k$ and where:

$$
\Upsilon(a, b, u, v)=\int_{0}^{+\infty} \frac{(z+a)(z+b)}{\pi(u v)^{3 / 2}} \exp \left(-\frac{(z+a)^{2}}{2 v}\right) \exp \left(-\frac{(z+b)^{2}}{2 u}\right) d z
$$

To sum up, the computation of $C^{+}$, an up and in cumulative Parisian call option can be done by means of a triple quadrature. The first quadrature yields $\Upsilon$ whilst the second and third ones give $\xi$ and therefore $C^{+}$thanks to formula (9). It is at this point that formula (8) intervenes and gives the price of a down and in cumulative Parisian put option. Finally, as in Section 3, the general parity relationship (6) provides the down and out put price. This allows computing directly the consortium and Government cumulative Parisian guarantees.

\subsection{Qualitative results}

We plot in figures 9 and 10 the values of the Government and consortium guarantees. We observe that the cumulative Parisian Government guarantee is more expensive than the standard Parisian one. As for the cumulative Parisian consortium guarantee, it admits the opposite behavior. These features are justified by the fact that exclusion is more probable under a cumulative Parisian covenant (when choosing the same window $d$ and same threshold $L$ ).

It thus appears that the introduction of a cumulative Parisian covenant has the same impact on Government and consortium guarantees than the introduction of a standard Parisian covenant. The effects are just even more pronounced: the Government guarantee value is extremely high whilst the consortium one is very low. This is equivalent to a subsidies effect from the Government and might not be as beneficial to the system as it looks like. In the

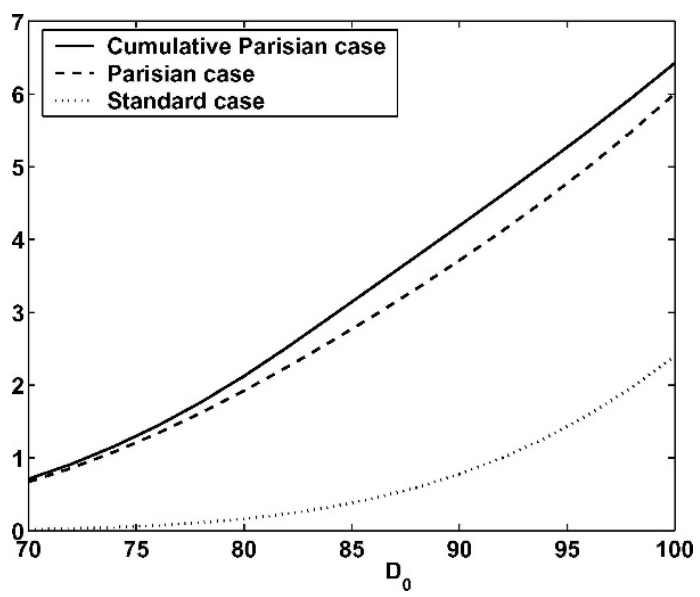

Figure 9. Government guarantee. 


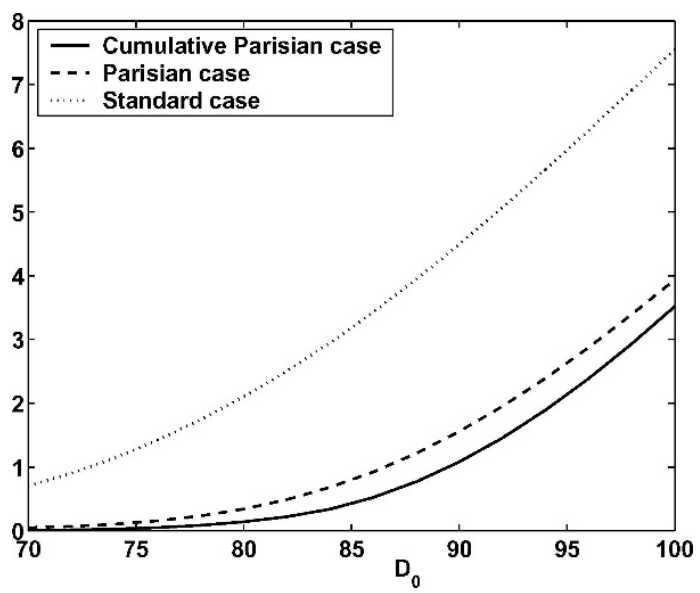

Figure 10. Consortium guarantee.

next section, we build a new type of covenant that has different implications in terms of minimization of risk.

\section{Design of a new type of covenant}

In the preceding section, we studied the impact of introducing Parisian and cumulative Parisian covenants in deposit guarantee contracts. Following these covenants, a bank remaining too long in distress is excluded from the consortium; the goal being to incite banks to reduce their risks. Yet, it appears that, in such a system, these covenants might lead to adverse bank behaviors-because the fair premia are only paid to the consortium, not to the Government in an Italian-style system. Exclusion would in fact be beneficial to banks and allow them to levy subsidies from the Government. It is therefore important to design a new type of covenant that can, indeed, make banks reduce their risks.

\subsection{Construction of the covenant}

Let us introduce the following covenant: when the assets of a bank under surveillance remain too low too long (in a standard Parisian fashion), then, at the end of the year, the bank has to pay a penalty to the consortium-but is not excluded from it. This type of penalty can be viewed as a kind of security loading payed ex-post; it can also be related to the penalty a European state like France or Germany should pay in case its deficits remain too high (as a percentage of GDP) for a given period.

The main goal of making banks pay a penalty at the end of the year if their assets have remained too low too long (typically one month) is not to overcharge them. The goal is in fact to incite them not to be in the situation of paying the penalty, in other words, to incite them to keep high levels of assets (with respect to total deposit amounts). The same kind of 
covenant could also concern the leverage: the consortium could impose banks to maintain low levels of leverage by asking them to pay a penalty if it remains too high too long.

From now on, we suppose that the covenant is written on the assets. A penalty $M$ is paid at the end of the year if the bank assets remain more than the time lag $d$ below the threshold $L$. The covenant from the point of view of the deposit institution thus corresponds to an additional fee paid at the end of the year if it did not comply with its regulatory obligations. This covenant or ex post security loading, can be priced by standard optional methodology, through the formula:

$$
E_{Q}\left(e^{-r T} M \mathbb{1}^{P}\right)
$$

where $\mathbb{1}^{p}$ is equal to one in case of no regulatory compliance. This formula corresponds to the one of a simple Parisian digital option; we define this type of option and show how to compute it in the next subsection.

\subsection{Pricing Parisian digital options}

Now, define a Parisian digital option or $P D G$ as follows: the holder of the option receives a given amount $M$ provided the underlying verifies a standard Parisian condition. Such a product admits a simple valuation formula:

$$
P D G(T)=E_{Q}\left(e^{-r T} M \mathbb{1}^{p}\right)=M e^{-r T} Q(\tau \leq T)
$$

where $\tau$ is the time when the underlying $V$ has remained more than $d$ beyond the barrier $L$. We will obtain a semi-closed formula for the above probability, and this will enable us to price Parisian digital options or guarantees. This is the first place where, to our knowledge, a valuation formula is provided for these derivatives; the full proof is available from the authors upon request.

When $V_{0} \geq L$, one shows that:

$$
Q(\tau \leq T)=e^{-\frac{m^{2} T}{2}}\left(\int_{-\infty}^{l} h_{2}(T, y) e^{m y} d y+\int_{l}^{+\infty} h_{1}(T, y) e^{m y} d y\right)
$$

where $h_{1}(T, y)$ and $h_{2}(T, y)$ are both known through their Laplace transforms $\hat{h}_{1}$ and $\hat{h}_{2}$. $\hat{h}_{2}$ is given in Section 3.2 and $\hat{h}_{1}$ writes as:

$$
\hat{h}_{1}(\lambda, y)=\frac{e^{(2 l-y) \sqrt{2 \lambda}} \Psi(-\sqrt{2 \lambda d})}{\sqrt{2 \lambda} \Psi(\sqrt{2 \lambda d})}
$$

where $l=\frac{1}{\sigma} \ln \left(\frac{L}{x}\right)$ and $m=\frac{1}{\sigma}\left(r-q-\frac{\sigma^{2}}{2}\right)$.

To value a $P D G$, Parisian digital option or guarantee, one needs to compute formula (12). This can be achieved by means of inverse Laplace transforms (giving $h_{1}$ and $h_{2}$ ) followed by quadratures. 


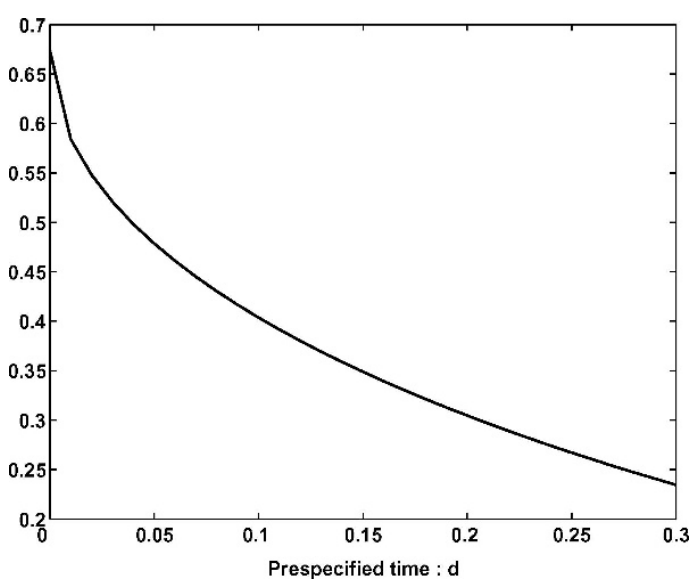

Figure 11. Covenant value w.r.t. the window.

\subsection{Impact of the new covenant}

Let us now study the impact of introducing a Parisian digital covenant. We start by choosing the following values for the parameters:

\begin{tabular}{ccccc}
\hline$V_{0}$ & $r$ & $\theta$ & $T$ & $D_{0}$ \\
\hline 100 & 0.03 & 1 & 1 & 70 \\
\hline
\end{tabular}

In figure 11 we plot the value of the covenant for a window $d$ ranging from 0 to 0.3 year, $L=90$ and $\sigma=0.25$. We can observe that for high values of the window, the covenant value is low. This is because when $d$ is high, the probability that the bank has to pay a penalty is low, and therefore the value of the covenant to the consortium is low.

Then, in figure 12, we graph the dependence of the covenant value with respect to the threshold $L$. We make it range between the initial value of the deposits $D_{0}$ and the initial value of the assets $V_{0}$, that is between 70 and 100. As concerns the assets volatility, we keep $\sigma=0.25$; the window $d$ is set to one month. For high levels of $L$, a penalty payment becomes much likely, which results in a higher value of the covenant for the consortium-as can be seen from this plot.

Let us conclude this section by an analysis of figure 13 which displays the dependence of the covenant value with respect to the assets volatility. We choose for this graph a threshold $L=90$; the assets volatility varies between 5 and $30 \%$ and each curve corresponds to a different value of the window ( $d$ varying from one week to six months).

The higher the assets volatility, the higher the likelihood of a penalty and therefore the higher the value of the covenant for the consortium. This is exactly what we aimed at 


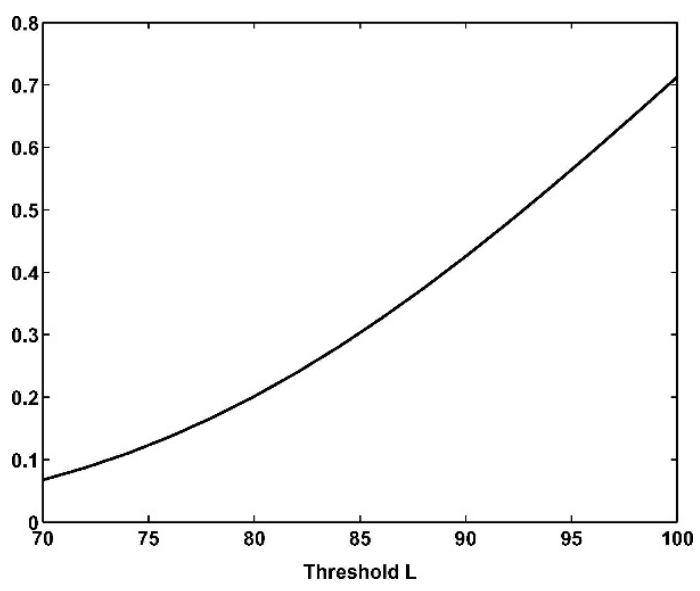

Figure 12. Covenant value w.r.t. the threshold.

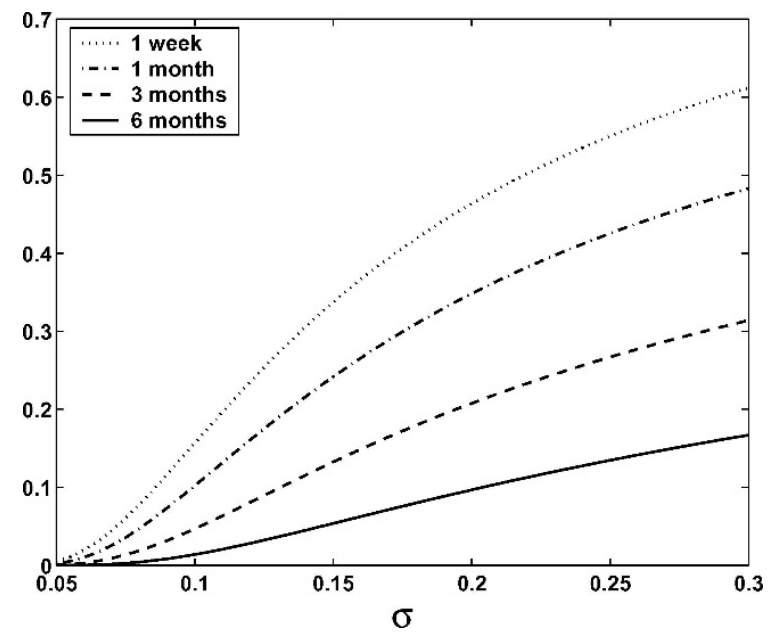

Figure 13. Covenant value w.r.t. the assets volatility.

constructing: this type of covenant should incite bank to reduce their risks by reducing the volatility of their assets. Of course a short window increases the value of the covenant and corresponds to a more stringent surveillance of banks. Similarly, a covenant written on the leverage would incite bank to improve their liability structure.

\section{Conclusion}

This article studied bank deposits insured by a consortium, as is the case in Italy. We assumed that each bank is insured up to a given quantity $K$ by the consortium. If the bank defaults of 
more than $K$, the Government takes care of the exceeding losses. We priced the consortium and Government guarantees in a Merton-like model.

A way to induce banks to reduce their risks would be to exclude them from the consortium when their assets remain too low too long. Designing of such covenants yields to an increase of the Government guarantee with respect to the consortium one. Monitoring of the leverage instead of the assets would give the same results. In practice, one can conceive that it is not at all a good idea because, in such a system (like the Italian one), banks do not pay premia to the Government but only to the consortium. Such covenants incite banks to levy subsidies from the Government and to, indeed, increase their risks.

To remedy this misplaced feature, we designed a new type of covenant. Our suggestion is the following: the consortium should impose penalties to the banks which did not comply with a regulatory provision on their assets or leverage. This provision can be of a Parisian type. The assets of a bank should not stay more than a given period below a given level; by analogy, one could ask the leverage not to stay more than a given time span above a given threshold.

\section{References}

BERNARD, C., LE COURTOIS, O., and QUITTARD-PINON, F. [2005]: "A New Procedure for Pricing Parisian Options," The Journal of Derivatives, 12(4), 45-53.

BLACK, F., and SCHOLES, M. [1973]: "The Pricing of Options and Corporate Liabilities," The Journal of Political Economy, 81(3), 673-654.

CHAN, Y.-S., GREENBAUM, S.I., and THAKOR, A.V. [1992]: "Is Fairly Priced Deposit Insurance Possible?" The Journal of Finance, 47(1), 227-245.

CHESNEY, M., JEANBLANC, M., and YOR, M. [1997]: "Brownian Excursions and Parisian Barrier Options," Advances in Applied Probability, 29, 165-184.

DE GIULI, M.E., MAGGI, M.A., and PARIS, F.M. [2003]: "Pricing Mutual Bank Deposit Guarantees,” Working Paper, 10th Annual Conference of the Multinational Finance Society, Montreal, Canada, paper MCF-136.

FEDERAL DEPOSIT INSURANCE CORPORATION [2000]: "Deposit Insurance Options Paper," FDIC Publications, pp. 1-87.

HABER, R., SCHÖUNBUCHER, P., and WILMOTT, P. [1999]: "Pricing Parisian Options," Journal of Derivatives, 6(3), 71-79.

HUGONNIER, J. [1999]: “The Feynman-Kac Formula and Pricing Occupation Time Derivatives," International Journal of Theoretical and Applied Finance, 2(2), 153-178.

LAEVEN, L. [2002]: "International Evidence on the Value of Deposit Insurance," The Quaterly Review of Economics and Finance, 42, 721-732.

MERTON, R.C. [1974]: "On the Pricing of Corporate Debt: The Risk Structure of Interest Rates," Journal of Finance, 29, 449-470.

MERTON, R.C. [1977]: "An Analytic Derivation of the Cost of Deposit Insurance and Loan Guarantees," Journal of Banking and Finance, 1, 3-11.

MERTON, R.C. [1978]: "On the Cost of Deposit Insurance When There are Surveillance Costs," Journal of Business, 51, 439-452.

MORAUX, F. [2002]: "On Cumulative Parisian Options," Finance, 23, 127-132.

RONN, E., AND A. VERMA [1986]: "Pricing Risk-Adjusted Deposit Insurance," The Journal of Finance, 41, 871-895.

SHIBUT, L. [2002]: “Should Bank Liability Structure Influence Deposit Insurance Pricing?" FDIC Working Paper No. 2002-01. 